\title{
On the computations of decay widths of Fano resonances
}

\author{
T. Miteva, ${ }^{1, *}$ S. Kazandjian, ${ }^{1}$ and N. Sisourat ${ }^{1}$ \\ ${ }^{1}$ Sorbonne Universités, UPMC Univ Paris 06, UMR 7614, \\ Laboratoire de Chimie Physique Matière et Rayonnement, F-75005 Paris, France
}

(Dated: June 16, 2016)

\begin{abstract}
In this paper we present an $a b$ initio approach to the computation of decay widths of Fano resonances. The method relies on Fano theory, in which a resonance is described as a bound state embedded in and interacting with a continuum of states. In our approach, we use the Configuration Interaction (CI) method to describe the bound-like and continuum-like parts of the resonance wave function. The aim of this Fano-CI method is to provide decay widths of resonances at a low computational cost such that large systems can be treated. Along with the implementation of the method, we present benchmark calculations of decay widths of Auger and ICD processes in Ne atom, and $\mathrm{Ne}_{2}$ and NeAr dimers. Our results are in good agreement with the decay widths from other theoretical and experimental works. This makes the Fano-CI approach a promising method for the treatment of Fano resonances.
\end{abstract}

*Electronic address: tsveta.miteva@upmc.fr 


\section{INTRODUCTION}

Resonances are metastable states, which decay in time into several subsystems. Among the different types of resonances, a Fano (also called Feshbach) resonance is described as a bound state embedded in one or more continua of an unperturbed Hamiltonian. The coupling due to the full Hamiltonian then turns the bound state into a metastable one, which as time passes breaks into two or more products [1-3]. A clear demonstration of this resonance scattering phenomenon in atomic and molecular physics is the Auger effect [4]: after core ionisation of an atom or a molecule an outer valence electron of this excited ion fills the inner vacancy and the excess energy is used to eject a secondary electron (socalled Auger electron). The Auger effect in atoms has been thoroughly investigated for more than four decades (see [5] and references therein). However, studying the Auger effect in molecules and clusters remains challenging owing to the lack of spherical symmetry and the extra vibrational degrees of freedom.

In this context, Lenz Cederbaum and co-workers have made outstanding contributions to the development of Auger theory, its understanding as well as the computational treatment of this effect in molecules. A major contribution is the elaboration of the so-called Algebraic Diagrammatic Construction (ADC) method [6] (see [7] for a recent review), which allows for a direct and efficient access to the ionisation energies and spectral intensities for small to moderate size molecules. Using this method for doubly ionised molecules combined with a population analysis technique $[8,9]$, they have shown that Auger spectra of molecules can be accurately computed, thus providing a powerful theoretical tool for the routine simulation and interpretation of the spectra of polyatomic systems. Another important aspect in the theoretical description of the Auger process is its lifetime, which is typically in the femtosecond time scale, and thus the process competes with the vibrational motion of light atoms within molecules. The nuclear dynamics must therefore be accounted for in order to provide accurate simulation of Auger processes. To this end, Lenz Cederbaum and co-workers have derived a full time-dependent quantum approach $[10,11]$ which allows for the computation of experimental observables such as Auger electron distribution or kinetic energy of the ionic fragments formed after the Auger decay (the so-called kinetic-energy-release, KER, spectrum). Furthermore, this time-dependent approach is the starting point of other approximate methods that can be used to investigate large systems [12]. 
Lenz Cederbaum and co-workers made another eminent contribution in the study of resonance phenomena in atomic and molecular systems. They discovered a whole new unsuspected kind of non-radiative decay processes, opening new perspectives in physics, chemistry and recently in biology. It started in 1997 when they revealed that an inner valence ionised molecule can release its excess energy by transferring it to a neighbouring molecule which is then ionised. This process was named Intermolecular Coulombic Decay (ICD) [13]. Since this pioneer work, ICD was shown to be a general decay mechanism for highly excited ions, atoms or molecules embedded in a chemical environment [14]. Following this discovery, Cederbaum and co-workers uncovered other related interatomic and intermolecular decay processes such as Electron Transfer Mediated Decay (ETMD) [15] or Interatomic Coulombic Electron Capture (ICEC) [16]. Beyond their fundamental importance, all these processes have attracted considerable and rising attention for several reasons. First, these processes are direct sources of low energy electrons and radical cations both of which play a central role in radiation chemistry and radiobiology (some examples of their importance in this context can be found in [17-20]). Furthermore, the processes generally involve the participation of the nearest neighbours and are strongly influenced by the distance and the orientation of the two systems. These properties may be used to probe the close chemical environment of a selected molecule, paving the way for original analytical tools.

An important characteristic of all these resonance phenomena is their energy width, or turning from the energy to the time domain, their lifetime. Accurate calculations of decay widths are a difficult task since one has to deal with both the many-body and the scattering nature of the problem. Various approaches have been developed for the accurate computations of decay widths of rather small systems [21-24]. More approximate techniques relying on electron population analysis have been devised for larger systems [25]. Several methods specifically designed for the calculations of (both intra- and intermolecular) decay widths of moderate size systems have been developed by Lenz Cederbaum and co-workers. The first one is the Complex Absorbing Potential (CAP) method. The idea underlying the CAP method is to introduce an absorbing boundary condition in the exterior region of the system by an analytical continuation of the Hamiltonian, which makes the wave function of the scattered electron square integrable, i.e. representable in a $\mathcal{L}^{2}$ basis. The CAP method has been used in combination with CI [26], ADC [27] and Equation-of-Motion CoupledCluster (EOM-CC) $[28,29]$ methods. The second approach relies on the Fano theory of 
resonances combined with the Stieltjes imaging technique [30, 31]. The decaying state is described as a discrete bound state embedded in and interacting with a continuum of states. The bound state and an approximation of the continuum states are obtained from electronic structure methods. The Stieltjes imaging procedure is finally used to recover the correct normalisation of the continuum states. The simplest approach within this framework is the Wigner-Weisskopf method [30], which is based on the lowest order perturbation theory. In this approach, the decaying and final electronic states are obtained using the restricted Hartree-Fock solution of the neutral systems. Relaxation and correlation effects are therefore neglected which makes the method only a semi-quantitative one. A more accurate approach relies on the ADC technique [31]. The bound state and an approximation to the continuum states are obtained from ADC calculations which effectively treat the electronic correlation and relaxation effects.

In this work, we describe a method lying between the Wigner-Weisskopf and the FanoADC methods. Its aim is to provide a quantitative estimate of decay widths at low computational cost such that large molecular systems can be considered. Our approach relies on the Configuration Interaction (CI) technique in restricted active spaces and we thus called it Fano-CI. In this article, we present in detail the implementation of the method and discuss some important numerical considerations for the efficient calculations of decay widths. Finally, we benchmark the method against the available data on three well studied examples.

The paper is organised as follows. In the next section we outline the theoretical basis of the Fano-CI method. A derivation of the Fano-CI expressions is presented in Appendix A. In Sec. III we present benchmark calculations of decay widths of Auger and ICD processes following core ionisation of $\mathrm{Ne}$ atom and inner valence ionisation of $\mathrm{Ne}$ in $\mathrm{Ne}_{2}$ and $\mathrm{NeAr}$, respectively. The summary and conclusions are given in Sec. IV.

\section{METHODS}

For completeness, we briefly outline the Fano theory of resonances following the derivation of Howat et al. [5]. Next, we present and discuss the Fano-CI method. We then sketch the Stieltjes procedure needed to extract a continuous approximation to the decay width from a discrete representation of the continuum. Finally, we discuss the choice of basis set and Hartree-Fock reference in the calculation of Fano-CI decay widths. 


\section{A. Fano theory of resonances}

Similarly to the Fano-ADC method [31], the Fano-CI approach is devised for the treatment of Feshbach or Fano resonances. In the Fano formalism [1, 5], the wave function at energy $E$ in the vicinity of the resonance is represented as a superposition of a discrete component, $\Phi$, and continuum components, $\chi_{\beta, \epsilon}$, corresponding to the $N_{c}$ decay channels

$$
\Psi_{\alpha, E}=a_{\alpha}(E) \Phi+\sum_{\beta=1}^{N_{c}} \int d \epsilon C_{\beta, \epsilon}(E) \chi_{\beta, \epsilon}, \alpha=1, \ldots N_{c} .
$$

Here the index $\beta$ runs over the $N_{c}$ decay channels and $\epsilon$ is the kinetic energy of the outgoing electron. The bound part of the wave function, $\Phi$, is assumed to be an isolated resonance, not interacting with other resonances. It has a mean energy

$$
E_{\Phi}=\langle\Phi|\hat{H}| \Phi\rangle
$$

where $\hat{H}$ is the full electronic Hamiltonian of the system. The continuum states $\chi_{\beta, \epsilon}$, in their turn, are assumed to diagonalise the Hamiltonian

$$
\left\langle\chi_{\beta, \epsilon}|\hat{H}-E| \chi_{\beta^{\prime}, \epsilon^{\prime}}\right\rangle \approx \delta_{\beta, \beta^{\prime}} \delta\left(E_{\beta}-E_{\beta^{\prime}}+\epsilon-\epsilon^{\prime}\right)\left(E_{\beta}+\epsilon-E\right)
$$

and are thus also non-interacting.

Solving the Schrödinger equation $(\hat{H}-E) \Psi_{\alpha, E}=0$, one can determine the bound and continuum amplitudes, $a_{\alpha}(E)$ and $C_{\beta, \epsilon}(E)$, respectively [1, 5]. From the expression for $\left|a_{\alpha}(E)\right|^{2}[5]$, one obtains the relationship between partial $\Gamma_{\beta}$ and total widths

$$
\Gamma=\sum_{\beta}^{N_{c}} \Gamma_{\beta}=2 \pi \sum_{\beta}^{N_{c}}\left|\left\langle\Phi\left|\hat{H}-E_{r}\right| \chi_{\beta, \epsilon_{\beta}}\right\rangle\right|^{2}
$$

where $\epsilon_{\beta}$ is the asymptotic kinetic energy of the emitted electron and $E_{r}$ is the energy of the resonance. In practice, the energy of the resonance is approximated with the expectation value of the Hamiltonian with respect to the bound component of the resonance, i.e. $E_{r} \approx E_{\Phi}$ [31]. This approximation is justified provided that the shift of the resonance, resulting from the interaction with the continuum, is smaller than the error in the calculation of the energy of the bound state [31].

As can be seen from Eq. (4), the total decay width is a sum of the partial decay widths to different decay channels. The expression for the partial decay widths given in (4) is known as "single channel" approximation, where no interaction between the decay channels is assumed. 


\section{B. Fano-CI method}

The evaluation of expression (4) requires suitable approximations for the bound $(\Phi)$ and continuum $\left(\chi_{\beta, \epsilon_{\beta}}\right)$ parts of the resonance. In the following, we discuss the particular approximations made in the Fano-CI method. The discussion is focused on a singly ionised resonance of a closed shell atom or molecule. However, the method can be generalised to $n$-tuply ionised states, as well as ionisation satellites and excited states of both closed and open shell species.

Let us consider the specific case of an electronic decay process following single ionisation of an $N$-electron atom or molecule. In this case, the bound part of the resonance state is the initial, singly ionised state of the system, whereas the continuum components comprise all doubly ionised states with an electron in the continuum. Normal Auger decay, ICD and ETMD are examples of such electronic decay processes. Within the Fano-CI method, the initial singly ionised state is approximated as a one-hole (1h) configuration, i.e. an $(N-1)$ electron Slater determinant, where an electron is removed from orbital $\varphi_{i}$

$$
|\tilde{\Phi}\rangle=\left|\Psi_{i}\right\rangle=c_{i}\left|\Psi_{0}\right\rangle
$$

Here, $c_{i}$ indicates the annihilation operator and $\left|\Psi_{0}\right\rangle$ denotes the Hartree-Fock ground state of the system. The continuum states $\left|\chi_{\beta, \epsilon}\right\rangle$ are approximated as discrete square integrable states $\left|\tilde{\chi}_{q}^{a}\right\rangle$, which are linear combinations of two-hole-one-particle (2h1p) configurations $\left|\Psi_{a k l}\right\rangle$

$$
\left|\chi_{\beta, \epsilon}\right\rangle \approx\left|\tilde{\chi}_{q}^{a}\right\rangle=\sum_{k, l: \epsilon_{k, l}>\epsilon_{i}}^{n_{o c c}} C_{a k l}^{q}\left|\Psi_{a k l}\right\rangle
$$

Here, the indices $k, l$ and $a$ stand for occupied and virtual Hartree-Fock orbitals, respectively. The $2 \mathrm{~h} 1 \mathrm{p}$ configurations $\left|\Psi_{a k l}\right\rangle$ are characterised with two holes in the space of Hartree-Fock states $\left\{\varphi_{k}\right\}$ of energy $\epsilon_{k}>\epsilon_{i}$, and a particle in $\varphi_{a}$. Note that in our implementation all 2h1p configurations entering the expansion of a given final state $\left|\tilde{\chi}_{q}^{a}\right\rangle$, have a fixed virtual orbital, $\varphi_{a}$.

The coefficients $C_{a k l}^{q}$ are determined by solving the eigenvalue problem

$$
\hat{H}\left|\tilde{\chi}_{q}^{a}\right\rangle=E_{q}^{a}\left|\tilde{\chi}_{q}^{a}\right\rangle
$$

in the basis of the following doublet spin adapted configurations (or configuration state 
functions)

$$
\begin{aligned}
\left|\Psi_{a k l}^{S_{1}}\right\rangle & =\frac{1}{\sqrt{2}}\left(\bar{c}_{a}^{\dagger} c_{k} \bar{c}_{l}-\bar{c}_{a}^{\dagger} \bar{c}_{k} c_{l}\right)\left|\Psi_{0}\right\rangle \\
\left|\Psi_{a k k}^{S_{2}}\right\rangle & =\bar{c}_{a}^{\dagger} c_{k} \bar{c}_{k}\left|\Psi_{0}\right\rangle \\
\left|\Psi_{a k l}^{T}\right\rangle & =\frac{1}{\sqrt{6}}\left(\bar{c}_{a}^{\dagger} c_{k} \bar{c}_{l}+\bar{c}_{a}^{\dagger} \bar{c}_{k} c_{l}+2 c_{a}^{\dagger} c_{k} c_{l}\right)\left|\Psi_{0}\right\rangle
\end{aligned}
$$

Here, the superscripts denote the singlet $\left(S_{1,2}\right)$ and triplet $(T)$ spin states of the two holes. A bar over the creation $\left(c^{\dagger}\right)$ or annihilation $(c)$ operators stands for creation or annihilation of a spin orbital with spin $\beta$. A derivation of the expressions for the matrix elements of the Hamiltonian in the basis set of spin adapted configurations is given in Appendix A. The size of the resulting CI matrix is of the order of $n_{o c c}^{2}$, where $n_{o c c}$ is the number of occupied Hartree-Fock orbitals. In order to obtain all final states $\left|\tilde{\chi}_{q}^{a}\right\rangle$, one needs to solve the eigenvalue problem $(7) n_{\text {virt }}$ times, where $n_{\text {virt }}$ is the number of virtual orbitals. The multiple diagonalisation of a small matrix is a substantial reduction in the computational effort compared to, for example, the effort needed to diagonalise a single $\mathrm{ADC}(2 \mathrm{e})$ matrix, where the size of the $2 \mathrm{~h} 1 \mathrm{p}$ block is of the order of $n_{\text {occ }}^{2} n_{v i r t}$. The cost for the substantial reduction in the size of the Hamiltonian matrix in the Fano-CI method is that the coupling between the pseudocontinuum final states $\left|\tilde{\chi}_{q}^{a}\right\rangle$ is completely neglected. The $2 \mathrm{~h} 1 \mathrm{p}$ configurations are however partially coupled through Eq. (6).

Finally, the states $\left|\tilde{\chi}_{q}^{a}\right\rangle$ are used to compute the coupling matrix elements

$$
\gamma_{q}^{a}=2 \pi\left|\left\langle\tilde{\Phi}|\hat{H}-E| \tilde{\chi}_{q}^{a}\right\rangle\right|^{2}
$$

These coupling matrix elements cannot be directly used in Eq. (4) to compute the decay width. However, the total decay width can be recovered from them employing the Stieltjes procedure described below.

\section{Stieltjes procedure}

The states $\left|\tilde{\chi}_{q}^{a}\right\rangle$ computed using the Fano-CI method (Eq. (6)) cannot be directly associated with the true continuum wave functions. First of all, these states are normalised to unity rather than energy normalised, and as such they do not satisfy the appropriate scattering boundary conditions. Moreover, owing to the finite basis sets employed in practical calculations, these final states do not satisfy the energy conservation condition for a 
non-radiative decay process

$$
E_{\beta}+\epsilon_{\beta}=E_{r}
$$

except by a coincidence.

These difficulties can be resolved by applying a mathematical approach known as the Stieltjes imaging technique [32-34]. It relies on the fact that even though the square integrable $\left(\mathcal{L}^{2}\right)$ pseudocontinuum states cannot be used to compute the decay width directly, the spectral moments obtained from them are good approximations to the true spectral moments of the decay width.

$$
S(k)=\sum_{\beta=1}^{N_{c}} \int d E E^{k}\left|\left\langle\Phi\left|\hat{H}-E_{r}\right| \chi_{\beta, \epsilon_{\beta}}\right\rangle\right|^{2} \approx \sum_{q} \sum_{a} E_{q}^{k}\left|\left\langle\tilde{\Phi}\left|\hat{H}-E_{r}\right| \tilde{\chi}_{q}^{a}\right\rangle\right|^{2}
$$

The moment theoretical approach is based on the observation that the pseudocontinuum $\mathcal{L}^{2}$ wave functions approach the behaviour of the true continuum wave functions in the molecular interaction region provided that the basis set is sufficiently large to ensure a high density of pseudocontinuum states around the resonance energy. Usually, a series of calculations with an increasing number of spectral moments $S(k)$ is performed until a consistent result is obtained [31-34].

In practice it is difficult to obtain converged results without requiring a prohibitively large basis set. A question thus arises in the choice of the maximal Stieltjes order to be employed. In principle, higher orders provide more accurate results. However, owing to the finite basis sets, high order moments become inaccurate [34]. In the implementation of Fano-ADC method in Ref. [31], for example, the decay width is taken to be the average over a range of Stieltjes orders, for which it does not vary substantially. In this work, we computed the decay widths as averages over several Stieltjes orders, between 15 and 30

$$
\bar{\Gamma}=\frac{1}{15} \sum_{n_{S}=15}^{30} \Gamma\left(n_{S}\right)
$$

and we present the results with the respective standard deviation from this average value. Since the Fano-CI method is devised for large systems, this naturally imposes a limit on the sizes of the basis sets used. The use of small basis sets, however, results in a non-stationary behaviour of the computed decay width as a function of the Stieltjes order. By computing the standard deviation for each averaged decay width (Eq. (14)), we can give an estimate of the error resulting from the Stieltjes imaging procedure. As shown below, this error is small even for moderate basis set size. 


\section{Choice of basis sets and Hartree-Fock reference}

The choice of basis set is crucial for the accurate calculation of decay widths as the basis set has to provide a reliable description of both the discrete state and the continuum region of interest. This is usually achieved by employing large Gaussian basis sets (see e.g. $[20,31,35-39])$, which are a combination of the standard basis sets and diffuse or compact basis functions centred around the atom or molecule. In the case of ICD due to the low energy of the emitted electrons, the most commonly used basis sets include the standard Gaussian basis sets augmented with a large number of diffuse basis functions specifically designed for the description of Rydberg and continuum states [40]. In contrast, the Auger electrons are much faster and in order to describe them the standard Gaussian basis sets are often uncontracted or augmented with compact basis functions [20, 31], which ensures a non-zero density of states in the high energy region of the continuum. In our benchmark calculations of ICD widths we augmented the standard Gaussian basis sets with diffuse functions of the Kaufmann-Baumeister-Jungen (KBJ) type [40] on the atomic centres. In the case of Auger decay, we adopted a different approach: instead of uncontracting the basis set, we augmented it with sets of compact even tempered basis functions.

To construct the bound and continuum parts of the resonance state in the Fano-CI method, one needs to provide Hartree-Fock orbital energies and two-electron integrals as input. In the case of a singly ionised decaying state considered here, the ionisation step is accompanied by orbital relaxation effects, which are not accounted for in the Hartree-Fock calculation on the neutral system. These effects can be included in two ways. First, one can perform a restricted open-shell Hartree-Fock (ROHF) calculation, in which one can force an electron to be removed from the Hartree-Fock orbital of interest. Subsequently, the orbital energies and two-electron integrals generated in the ROHF step can be used in the Fano-CI procedure described in Sec. II B and Appendix A. Even though the expressions in Appendix A are not the rigorous analytical expressions for a ROHF reference function, the obtained decay widths are in good agreement with other theoretical results as we show later (see Sec. III).

The second way to account for orbital relaxation effects is to employ the so-called core equivalent or $(Z+1)$ approximation. It relies on the assumption that valence electrons are affected by the ionisation of a core electron in the same way as they would be if a proton 
is added to the nucleus. This approximation is justified only for core ionised states and is known to give fairly accurate estimates of core electron binding energies [41, 42]. Using this approximation, the Hartree-Fock orbital energies and two-electron integrals needed to compute the decay width of a core ionised state of an atom with atomic charge $Z$ are obtained from a closed shell calculation on the singly ionised atom with atomic charge $(Z+1)$. As we show in the next section, the decay widths obtained using the core equivalent approximation are in good agreement with other works.

\section{RESULTS AND DISCUSSION}

In this section, we evaluate the accuracy of the Fano-CI method by presenting benchmark calculations of electronic decay widths. Since the Fano-CI method is a general one, it can be applied both to intraatomic processes, such as the Auger decay, and to interatomic processes, such as ICD and ETMD. Therefore, we test the accuracy of the method by computing decay widths for the Auger and ICD processes, and comparing our results with available literature values. We chose the $\mathrm{Ne}^{+}\left(1 s^{-1}\right)$ resonance in an isolated $\mathrm{Ne}$ atom, and the $\mathrm{Ne}^{+}\left(2 s^{-1}\right)$ resonance in $\mathrm{Ne}_{2}$ and $\mathrm{NeAr}$ dimers as our benchmark cases. This choice was incited by the existence of extensive theoretical and experimental studies on the processes following core and inner valence ionisation of $\mathrm{Ne}[15,26,43-51]$.

The aim of our benchmark calculations is to compare the accuracy of the Fano-CI method with other methods for the computation of decay widths, in particular, the Fano-ADC(2e) method. Moreover, since the Fano-CI method is primarily intended to describe resonances in large systems for which basis sets of limited size must be employed, a thorough investigation of the dependence of the widths with respect to the basis sets was performed.

\section{A. Auger decay in $\mathrm{Ne}$ atom}

Let us first consider the decay of the $1 s^{1} 2 s^{2} 2 p^{6}{ }^{2} S$ state of $\mathrm{Ne}^{+}$. The only possible nonradiative relaxation pathway in this case is the KLL Auger decay, and, consequently, the total non-radiative decay width corresponds to the Auger width. The Auger decay leads to the population of the doubly ionised $2 s^{-2}{ }^{1} S, 2 s^{-1} 2 p^{-1}{ }^{3} P, 2 s^{-1} 2 p^{-1}{ }^{1} P, 2 p^{-2}{ }^{1} S, 2 p^{-2}{ }^{1} D$ states and to the ejection of an Auger electron of kinetic energy around $818 \mathrm{eV}$ [59]. The 


\begin{tabular}{lcccccc}
\hline \hline & \multicolumn{3}{c}{ Fano-CI } & \multirow{2}{*}{ Fano-ADC(2e) } & Other works \\
\cline { 2 - 4 } & & RHF & ROHF $(Z+1)$ & & \\
\hline \multirow{2}{*}{$\mathrm{Ne}^{+}\left(2 s^{-1}\right) \mathrm{Ne}$} & ${ }^{2} \Sigma_{g}^{+}$ & 5 & 6 & & $15^{a}$ & $10^{c}$ \\
& ${ }^{2} \Sigma_{u}^{+}$ & 8 & 6 & $15^{d}$ & $22^{e}, 19^{f}$ \\
$\mathrm{Ne}^{+}\left(2 s^{-1}\right) \mathrm{Ar}$ & 19 & 16 & & $251^{g}$ & $220 \pm 30^{h}$ \\
$\mathrm{Ne}^{+}\left(1 s^{-1}\right)$ & 152 & 298 & 199 & & \\
\hline \hline
\end{tabular}

${ }^{a} \mathrm{CAP} / \mathrm{CI}$, Ref. [52]

${ }^{b}$ Ref. [43]

${ }^{c} \mathrm{CAP} / \mathrm{CI}$ method, [26]

${ }^{d}$ Ref. [44]

${ }^{e}$ Wigner-Weisskopf theory, Ref. [15]

${ }^{f}$ Electron dynamics calculations, Ref. [46]

${ }^{g}$ Ref. [53]

${ }^{h}$ Experiment, Ref. [54, 55]

TABLE I: Comparison of the total Auger and ICD widths (meV) computed using the Fano-CI method with theoretical and experimental values for different benchmark systems $-\mathrm{Ne}_{2}, \mathrm{NeAr}$, Ne. In the case of $\mathrm{Ne}_{2}$ and $\mathrm{NeAr}$ the decay widths are taken at the equilibrium interatomic distances: $3.2 \AA$ and $3.5 \AA$, respectively. The ICD widths are computed using the cc-pVQZ basis set augmented with $7 s, 7 p$ and $7 d$ diffuse basis functions of the KBJ type in the case of $\mathrm{Ne}_{2}$ and $9 s, 9 p, 9 d$ functions in the case of $\operatorname{NeAr}[56,57]$. The Auger width of $\mathrm{Ne}\left(1 s^{-1}\right)$ is computed using the cc-pVQZ basis set [56] augmented with a set of $7 s, 7 p, 7 d$ even tempered basis functions.

decay width of the $\mathrm{Ne}^{+}\left(1 s^{-1}\right)$ resonance was computed following the procedure described in Sec. II. However, we also included the orbital relaxation effects as described in Sec. II D by performing a restricted open-shell HF calculation on the singly ionised atom (ROHF), where we forced an electron to be removed from the $1 s$ orbital, and a HF calculation on the closedshell $\mathrm{Na}^{+}$ion. The Hartree-Fock solution for the neutral and singly ionised systems was obtained using the GAMESS-US computational package [60], and the cc-pVDZ, cc-pVTZ and cc-pVQZ basis sets [61] augmented with $n$ sets of $s, p, d$ even tempered basis functions, where $n=1-7$ [58], thus ensuring sufficient density of states in the high energy part of the continuum.

The Hartree-Fock orbital energies and two-electron integrals were subsequently used to 


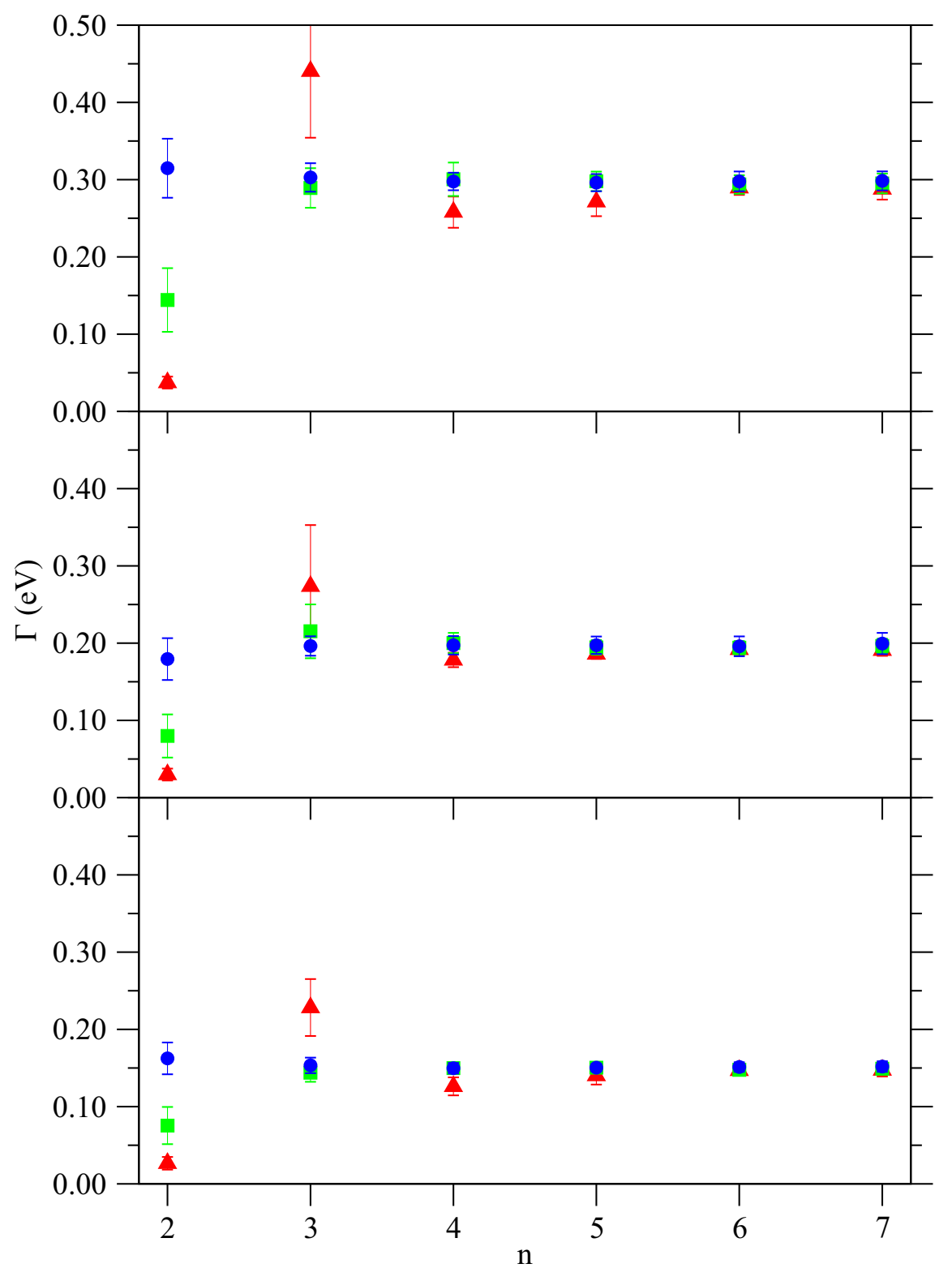

FIG. 1: Total Auger decay widths $(\mathrm{eV})$ of $\mathrm{Ne}^{+}\left(1 s^{-1}\right)$ computed using the Fano-CI method and the cc-pVDZ (red), cc-pVTZ (green) and cc-pVQZ (blue) basis sets augmented with additional sets of $n s, n p$ and $n d$ even tempered basis functions [58]. The decay widths shown in the lowermost panel were computed using the Hartree-Fock solution for the neutral atom (RHF). The middle panel shows the decay width computed employing the core equivalent approximation, i.e. the solution for $\mathrm{Na}^{+}$. Finally, the total Auger widths presented in the uppermost panel were computed from the Hartree-Fock solution for the singly ionised atom (ROHF).

compute the Auger decay width in the procedure described in Sec. II and Appendix A. The initial state is constructed as an $(N-1)$ Slater determinant, where an electron is removed 
from the $1 s$ orbital. Then we include the remaining occupied $2 s$ and $2 p$ orbitals in the space of one-particle states, out of which all possible two-hole combinations are formed. Subsequently, we construct and diagonalise the Hamiltonian matrix in the space of the $2 \mathrm{~h} 1 \mathrm{p}$ configurations as described in Sec. II B.

The results of the three different approaches to the computation of the Auger width are presented in Fig. 1. As the basis set increases, the Auger width of the $\mathrm{Ne}^{+}\left(1 s^{-1}\right)$ resonance converges to $152 \mathrm{meV}$ in the RHF case, to $199 \mathrm{meV}(Z+1)$ case and $298 \mathrm{meV}$ in the ROHF case. The Stieltjes errors are $7 \mathrm{meV}, 14 \mathrm{meV}$ and $13 \mathrm{meV}$, respectively, i.e. smaller than $10 \%$. The latter two decay widths, computed from the Hartree-Fock solutions for $\mathrm{Na}^{+}$and for the singly ionised Ne atom, are in better agreement with the experimental and theoretical natural linewidths of $220 \mathrm{meV}$ [54, 55] and $251 \mathrm{meV}$ [53], respectively (see Table I). Consequently, accounting for the orbital relaxation upon core ionisation, results in a more accurate decay width without increasing the computational overhead.

Fig. 1 also shows the behaviour of the computed decay width as a function of the number of additional sets of even tempered basis functions. A convergence of the Auger width with respect to the basis set augmentation is achieved already for $n=5$ for all considered basis sets (cc-pVXZ, X = D, T, Q). In the case of the largest basis set, cc-pVQZ, this result is even more pronounced as the convergence is observed already for $2 s, 2 p$ and $2 d$ compact Gaussian functions. Therefore, in the case of Auger decay, in order to obtain a satisfactory result for the decay width, one does not need a very high density of states in the continuum energy region of interest. This makes the Fano-CI method very promising in the treatment of large systems.

\section{B. ICD in $\mathrm{Ne}_{2}$ and NeAr}

Let us now consider the electronic decay processes following inner valence ionisation of $\mathrm{Ne}$ in $\mathrm{Ne}_{2}$ and NeAr. In this case, the intraatomic decay is energetically forbidden. Among the interatomic decay processes, ICD and ETMD are the only energetically allowed relaxation pathways $[15,48]$. The ETMD channel is open only in the NeAr dimer. Consequently, the total non-radiative decay width of the $\mathrm{Ne}^{+}\left(2 s^{-1}\right)$ vacancy corresponds to the ICD width in the case of $\mathrm{Ne}_{2}$ and to the sum of the ICD and ETMD widths in the case of NeAr. Note that according to Ref. [15], the ETMD contribution to the total decay width at the equi- 


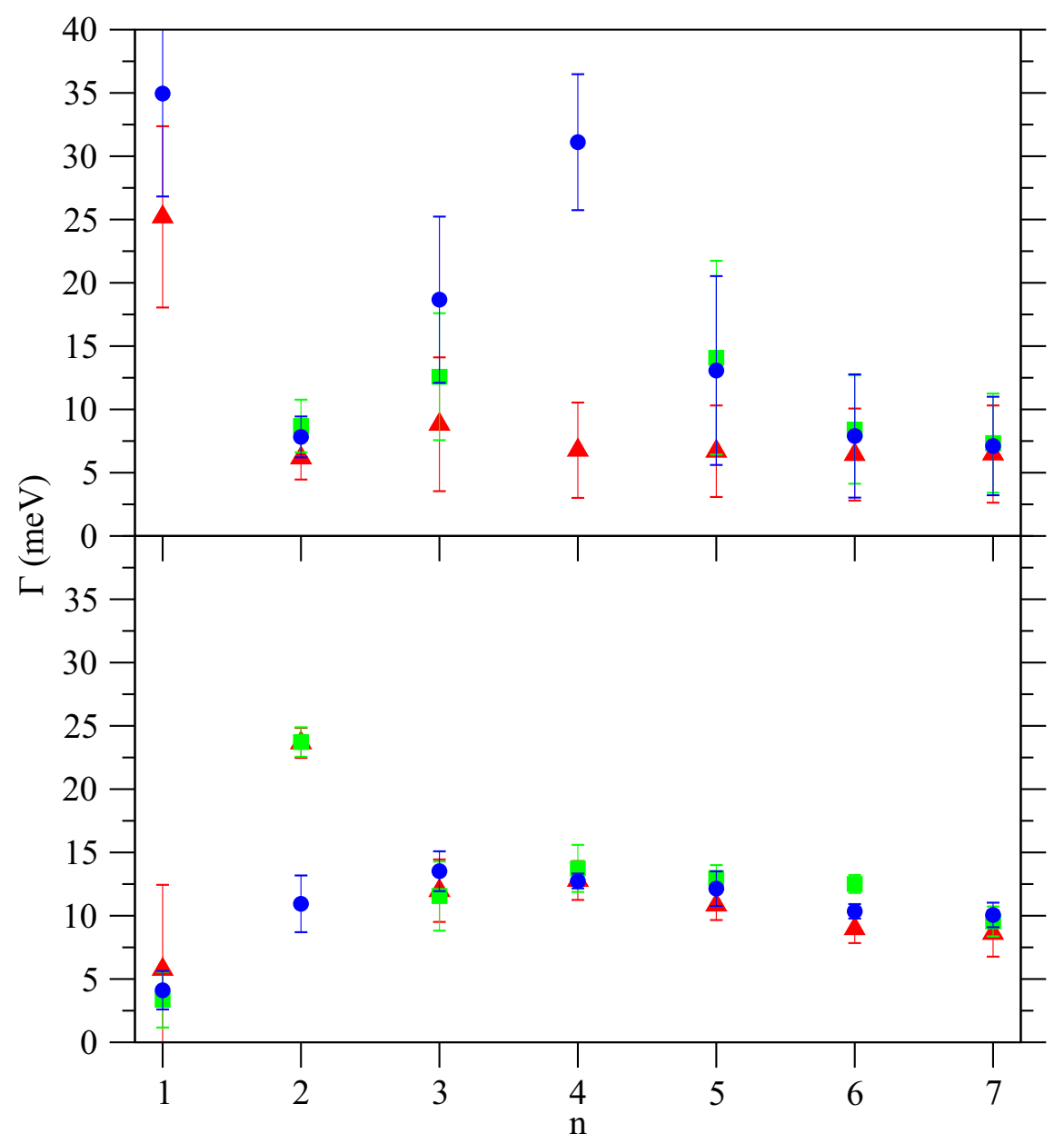

FIG. 2: Total decay width $(\mathrm{meV})$ of the $\mathrm{Ne}^{+}\left(2 s^{-1}\right) \mathrm{Ne} 2{ }^{2} \Sigma_{u}^{+}$state computed at the equilibrium internuclear distance $R_{e q}=3.09 \AA[62]$ using the Fano-CI method and the cc-pVDZ (red), ccpVTZ (green) and cc-pVQZ (blue) basis sets augmented with a set of $n s, n p$, nd diffuse KBJ functions. The decay widths in the lower panel were computed from the Hartree-Fock solution for the neutral dimer (RHF), whereas for the calculation of the decay widths in the upper panel, we employed the Hartree-Fock solution for the singly ionised dimer (ROHF).

librium interatomic distance is $\sim 10^{-4}$ smaller than ICD. The final states populated in the ICD process are two-site dicationic states - $\mathrm{Ne}^{+}\left(2 p^{-1}\right) \mathrm{Ne}^{+}\left(2 p^{-1}\right)$ and $\mathrm{Ne}^{+}\left(2 p^{-1}\right) \mathrm{Ar}^{+}\left(3 p^{-1}\right)$, whereas the final ETMD states in NeAr are characterised with two holes on the Ar atom: $\mathrm{NeAr}^{2+}\left(3 p^{-2}\right)$. The electrons emitted in the ICD process in $\mathrm{Ne}_{2}$ have energies between 0 and $2 \mathrm{eV}$ [51], whereas the electrons emitted in the decay of the $\mathrm{Ne}^{+}\left(2 s^{-1}\right) \mathrm{Ar}$ resonance, have energies between 3 and $9 \mathrm{eV}[15,45]$.

The Hartree-Fock solutions for both the neutral, and singly ionised dimers were obtained 


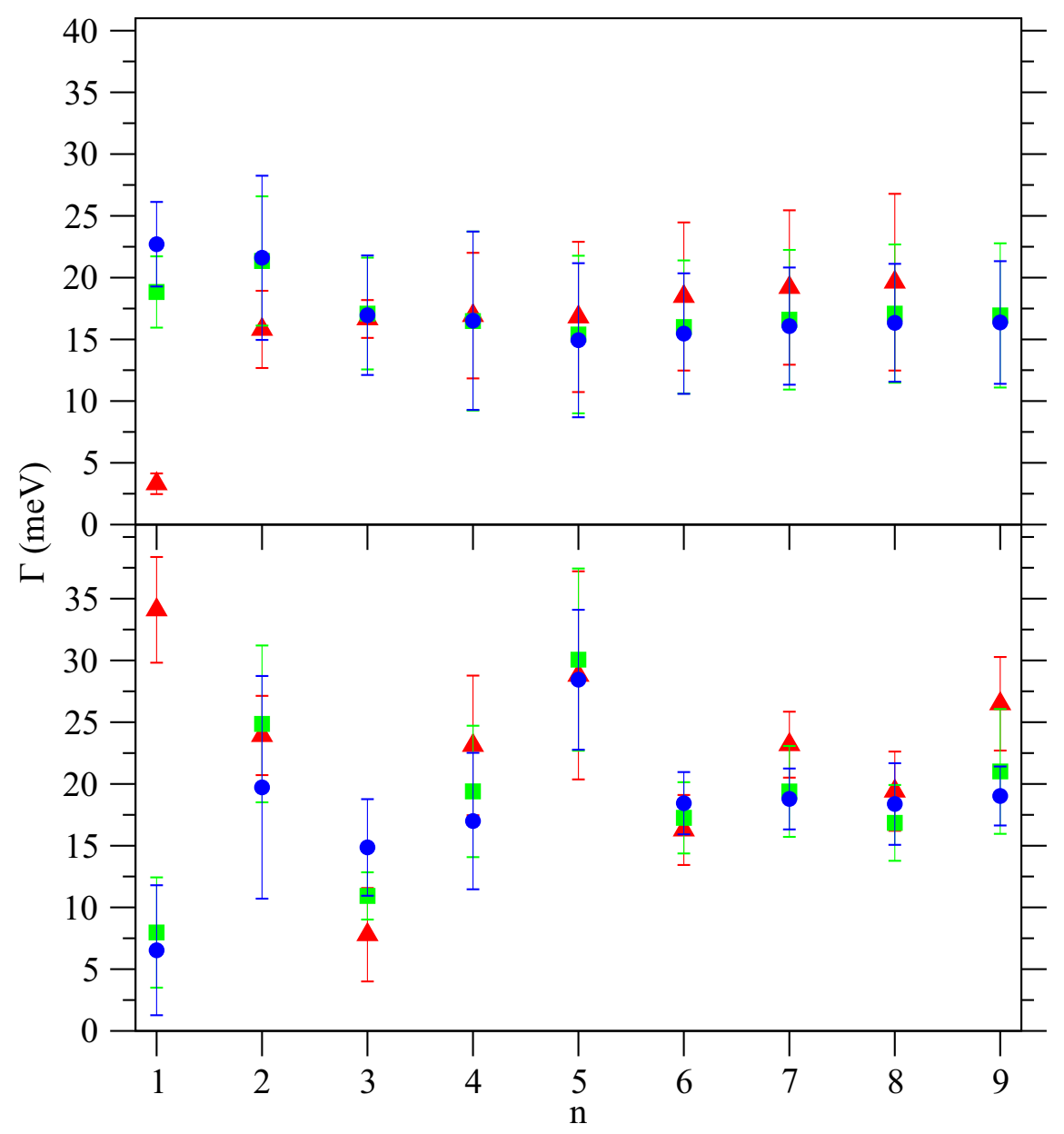

FIG. 3: Total decay width $(\mathrm{meV})$ of the $\mathrm{Ne}^{+}\left(2 s^{-1}\right) \mathrm{Ar}^{2} \Sigma^{+}$state computed at the equilibrium interatomic distance $R_{e q}=3.5 \AA \quad$ [63] using the Fano-CI method and the cc-pVDZ (red), cc-pVTZ (green) and cc-pVQZ (blue) basis sets augmented with a set of $n s, n p$, nd diffuse KBJ functions. The decay widths in the lower panel were computed from the Hartree-Fock solution for the neutral dimer (RHF), whereas for the calculation of the decay widths in the upper panel, we employed the Hartree-Fock solution for the singly ionised dimer (ROHF).

using the GAMESS-US computational package [60]. We used the cc-pVDZ, cc-pVTZ and cc-pVQZ [56, 57] basis sets on all atoms. The standard atomic basis sets were augmented with sets of diffuse $s, p$ and $d$ functions of the KBJ type [40]. The $(Z+1)$ approximation is not justified in the case of inner valence ionisation and was therefore not used here.

Then using the HF results, we followed the procedure described in Sec. II. The initial inner valence ionised state $\mathrm{Ne}^{+}\left(2 s^{-1}\right) \mathrm{X}(\mathrm{X}=\mathrm{Ne}$, Ar) was constructed as a $(N-1)$ Slater determinant, where an electron is removed from the Ne $2 s$ orbital. In Ne dimer, the $2 s$ 


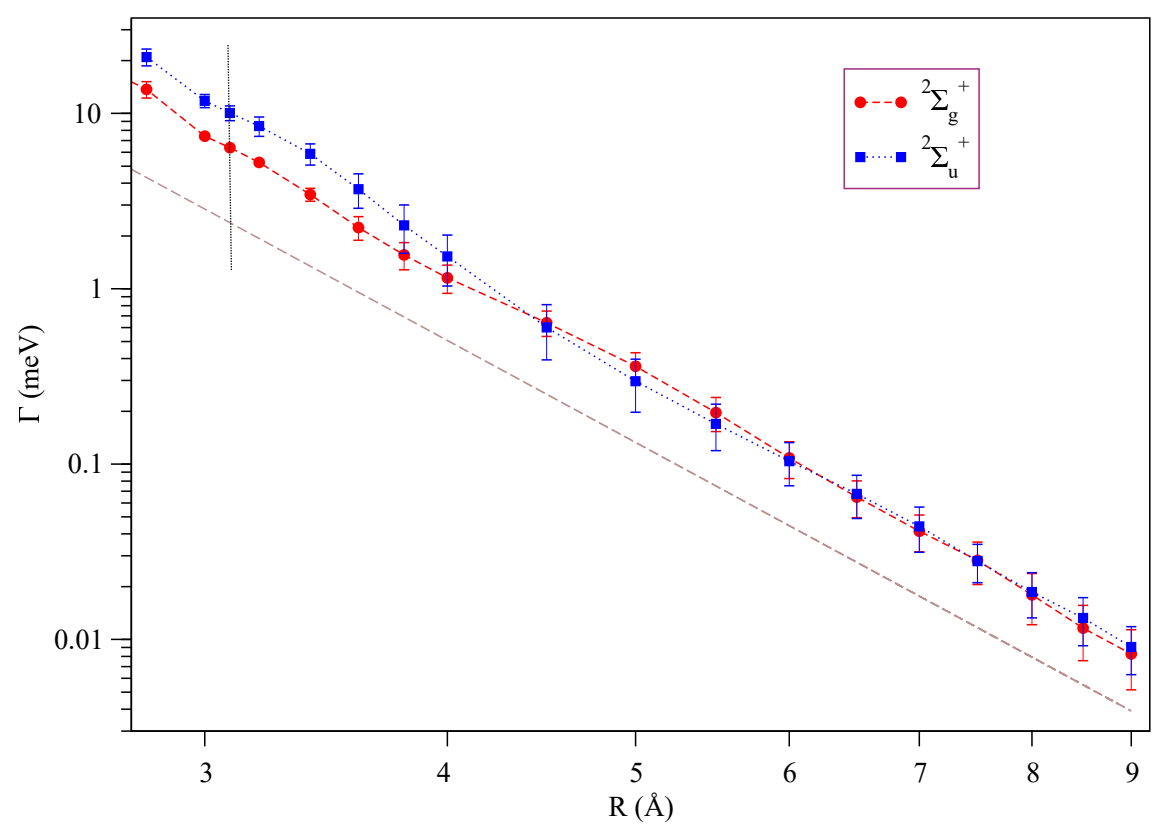

FIG. 4: Double logarithmic plot of the total non-radiative decay width of the $\mathrm{Ne}^{+}\left(2 s^{-1}\right) \mathrm{Ne} 2^{2} \Sigma_{g}^{+}$ (red circles) and $2^{2} \Sigma_{u}^{+}$(blue squares) states as a function of the internuclear distance $R$. The decay width was computed using the cc-pVQZ basis set augmented with $7 s, 7 p, 7 d$ diffuse KBJ functions. The dashed brown line is the $R$-dependent decay width obtained using the $1 / R^{6}$ approximation (15). The atomic data of Refs. [64] and [65] was used for the evaluation of Eq. 15. A vertical black line shows the ground state equilibrium interatomic distance, $R_{e q}=3.09 \AA$ [62]

vacancy is split into two states: a repulsive ${ }^{2} \Sigma_{g}^{+}$state and a bound ${ }^{2} \Sigma_{u}^{+}$state, both of which undergo ICD [48]. We computed the decay width for both of these states at the equilibrium distance $R_{e q}=3.09 \AA$ [62]. The one-particle states asymptotically corresponding to the Ne $2 p$ and $\operatorname{Ar} 3 p$ states were used to construct the final states of the decay processes: $\mathrm{Ne}^{+}\left(2 p^{-1}\right) \mathrm{Ne}^{+}\left(2 p^{-1}\right), \mathrm{Ne}^{+}\left(2 p^{-1}\right) \mathrm{Ar}^{+}\left(3 p^{-1}\right)$, and $\operatorname{NeAr}^{2+}\left(3 p^{-2}\right)$. Finally, the total decay widths were computed following the procedure described in Sec. II.

In Figs. 2 and 3 we present the total non-radiative decay widths of the $\mathrm{Ne}^{+}\left(2 s^{-1}\right) \mathrm{Ne}$ $2^{2} \Sigma_{u}^{+}$and $\mathrm{Ne}^{+}\left(2 s^{-1}\right) \mathrm{Ar}^{2} \Sigma^{+}$states, respectively, as a function of the basis set. Let us focus on the lower panels, which show the decay widths computed using the Hartree-Fock solution for the neutral dimers. As one can see, the decay widths converge to $10 \mathrm{meV}$ in the case of $\mathrm{Ne}_{2}$ and $19 \mathrm{meV}$ in the case of NeAr. The Stieltjes errors evaluated for the largest basis sets are, respectively, $1 \mathrm{meV}$ and $2 \mathrm{meV}$. An important distinction should be made between the two systems. Due to the lower ionisation potential of $\operatorname{Ar}(15.76 \mathrm{eV}$, compared to $21.56 \mathrm{eV}$ 
of $\mathrm{Ne}[66]$ ), the energy of the emitted ICD electron in NeAr is higher compared to $\mathrm{Ne}_{2}$ $[15,45,51]$. In order to cover the higher energy part of the continuum, one needs a larger Gaussian basis set. Consequently, as can be seen from Figs. 2 and 3, the decay width of the $\mathrm{Ne}^{+}\left(2 s^{-1}\right) \mathrm{Ne} 2^{2} \Sigma_{u}^{+}$state converges already upon the addition of a set of $7 s, 7 p, 7 d$ diffuse function, whereas in the case of the $\mathrm{Ne}^{+}\left(2 s^{-1}\right) \mathrm{Ar}^{2} \Sigma^{+}$state, one has to augment the basis sets further.

Let us now consider the upper panels of Figs. 2 and 3, where we show the basis set dependence of the total non-radiative decay widths of the $\mathrm{Ne}^{+}\left(2 s^{-1}\right) \mathrm{Ne} 2^{2} \Sigma_{u}^{+}$and $\mathrm{Ne}^{+}\left(2 s^{-1}\right) \mathrm{Ar}$ ${ }^{2} \Sigma^{+}$states computed using the HF calculation on the singly ionised dimers. The total nonradiative decay widths converge to $7 \mathrm{meV}$ in the case of $\mathrm{Ne}_{2}$ and to $16 \mathrm{meV}$ in the case of NeAr. Using a ROHF reference, results in a larger Stieltjes error: $4 \mathrm{meV}$ and $5 \mathrm{meV}$ for $\mathrm{Ne}_{2}$ and NeAr, respectively, for the largest basis sets. In the case of an interatomic decay process, initiated by inner valence ionisation, the relaxation effects are smaller compared to the core ionisation. Consequently, accounting for the orbital relaxation by computing the decay width using the Hartree-Fock solution for the singly ionised dimers, does not lead to a substantial improvement of the result. Unlike the Auger case, the decay width converges slower, and the Stieltjes error is larger $(\sim 50 \%$ in contrast to $\sim 4 \%$ in the case of Auger decay). This indicates that in the case of ICD one needs a high density of states in the low energy continuum, whereas to describe the Auger process, it is sufficient to have a few states in the high energy part of the continuum.

In Table I we present a comparison between the Fano-CI decay widths of the $\mathrm{Ne}^{+}\left(2 s^{-1}\right)$ resonance in $\mathrm{Ne}_{2}$ and $\mathrm{NeAr}$, and the values obtained in other theoretical works. The FanoCI decay widths are of the same order as those computed employing the Fano-ADC(2e) method. The Fano-ADC(2e) method is expected to be more accurate as it provides a better description of the initial state and fully accounts for the coupling between the final states. Furthermore, the Fano-ADC(2e) decay widths presented in Table I, were computed using large basis sets $[43,44]$. In the case of $\mathrm{Ne}_{2}$, the cc-pVQZ basis set was augmented with a set of $3 s, 3 p$, and $3 d$ Gaussian basis functions on each atom and 6 additional sets of $3 s, 3 p$, and $3 d$ Gaussians were distributed around the dimer [43], in the case of NeAr again the same basis set was used but the number of additional Gaussians was increased to $4 s, 4 p, 4 d$ [44]. The comparison between the Fano-CI and Fano-ADC $(2 \mathrm{e})$ results shows that accurate decay widths can be obtained with the "cheaper" Fano-CI method combined with moderate basis 
sets, which opens the possibility to investigate decay processes in large systems.

We tested the Fano-CI approach further by computing the ICD width as a function of the distance $R$ between the atoms in the neon dimer. The resulting $R$-dependent decay widths for both the $2^{2} \Sigma_{g}^{+}$and $2^{2} \Sigma_{u}^{+}$resonances are shown in Fig. 4. As expected for the interatomic decay process, the decay width decreases monotonically as the distance increases. At large distances, typically $R \geq R_{e q}$, the ICD width can be evaluated analytically with the virtual photon transfer mechanism [67-69]

$$
\Gamma(R)=\frac{3 \hbar}{3 \pi}\left(\frac{c}{\omega}\right)^{4} \frac{\tau_{r a d}^{-1} \sigma}{R^{6}}
$$

where $\tau_{\text {rad }}$ is the radiative lifetime of the Ne $2 s$ vacancy, $\sigma$ is the total ionisation cross section at the virtual photon energy $\hbar \omega$. In order to evaluate Eq. (15), we used the atomic data from references $[64,65]$. The virtual photon transfer result is also presented in Fig. 4. As can be seen from the figure, the $R$-dependent decay width computed using the Fano-CI method reproduces well the $1 / R^{6}$ dependence. However, similarly to the Fano-ADC $(2 \mathrm{e})$ method (see Ref. [43]), there is a discrepancy between the computed value and the one obtained by evaluating (15) at asymptotic distances.

\section{CONCLUSIONS}

In conclusion, we have presented a computationally efficient method for calculating decay widths of Fano resonances (such as in Auger, ICD and related processes). The method relies on the Fano theory of resonances which describes the resonance state as a discrete bound state embedded in and interacting with a continuum of states. Approximation of the bound and continuum parts are obtained from small CI calculations in restricted active spaces and with moderate basis set size. Benchmark examples show that the method provides accurate decay widths. The present method thus opens new perspectives in the study of Fano resonances in large atomic and molecular systems.

\section{ACKNOWLEDGEMENT}

This project has received funding from the Research Executive Agency (REA) under the European Union's Horizon 2020 research and innovation programme Grant agreement 
No 705515. N.S. acknowledges financial state aid managed by the Agence Nationale de la Recherche, as part of the programme Investissements d'avenir under the reference ANR-11IDEX-0004-02. 


\section{APPENDIX A: MATRIX AND COUPLING ELEMENTS}

All two-electron integrals are given in the physicists' notation.

In order to construct the final states, one needs to construct and diagonalise the Hamiltonian matrix in the basis of the possible $2 \mathrm{~h} 1 \mathrm{p}$ configurations for a fixed particle orbital. In the following, we will briefly outline this procedure as well as the computation of the coupling elements used in the Stieltjes method.

Let us consider the following partitioning of the Hamiltonian

$$
\begin{aligned}
\hat{H} & =\hat{H}_{0}+\hat{W}+\hat{V} \\
& =\sum_{p} \epsilon_{p} c_{p}^{\dagger} c_{p}+\sum_{p q} w_{p q} c_{p}^{\dagger} c_{q}+\frac{1}{2} \sum_{p q r s} V_{p q r s} c_{p}^{\dagger} c_{q}^{\dagger} c_{s} c_{r}
\end{aligned}
$$

where $\epsilon_{p}$ denote the Hartree-Fock one-electron energies, $V_{p q r s}$ denote the two-electron integrals in the basis of Hartree-Fock orbitals. The quantities $w_{p q}$, constituting the non-diagonal one-electron part, are expressed in terms of two-electron integrals

$$
w_{p q}=-\sum_{r} V_{p r[q r]} n_{r}
$$

where $n_{r}$ is the occupation number of the $r$ th orbital. Using the above notations, one can derive the matrix element between two $2 \mathrm{~h} 1 \mathrm{p}$ configurations in a general form (see Eq. A12 in $[70])$

$$
\begin{aligned}
\left\langle\Psi_{a k l}\left|\hat{H}-E_{0}\right| \Psi_{a^{\prime} k^{\prime} l^{\prime}}\right\rangle & =\delta_{a a^{\prime}} \delta_{k k^{\prime}} \delta_{l l^{\prime}}\left(\epsilon_{a}-\epsilon_{k}-\epsilon_{l}\right) \\
& +\delta_{a a^{\prime}} V_{k l\left[k^{\prime} l^{\prime}\right]} \\
& +\left(\delta_{k k^{\prime}} V_{a l^{\prime}\left[l a^{\prime}\right]}+\delta_{l l^{\prime}} V_{a k^{\prime}\left[k a^{\prime}\right]}\right)-(k \rightarrow l)
\end{aligned}
$$

where $a, a^{\prime}$ denote virtual and $k, l, k^{\prime}, l^{\prime}$ are occupied Hartree-Fock orbitals, respectively; $E_{0}$ is the ground state Hartree-Fock energy.

\begin{tabular}{|c|c|c|}
\hline $\mathbf{M}_{S_{1}}$ & $\mathbf{C}_{S_{1} S_{2}}$ & $\mathbf{C}_{S_{1} T}$ \\
\hline h.c. & $\mathbf{M}_{S_{2}}$ & $\mathbf{C}_{S_{2} T}$ \\
\hline h.c. & h.c. & $\mathbf{M}_{T}$ \\
\hline
\end{tabular}

FIG. 5: Block structure of the Hamiltonian matrix $\tilde{\mathbf{H}}$ in the basis set of doublet $2 \mathrm{~h} 1 \mathrm{p}$ spin eigenfunctions Eq. (8)-(10). The main and coupling blocks are denoted as $\mathbf{M}$ and $\mathbf{C}$, respectively. 
The Hamiltonian matrix in the basis of $2 \mathrm{~h} 1 \mathrm{p}$ spin eigenfunctions Eq. (8)-(10) is given in Fig. 5. From expression Eq. (A1) using the doublet 2h1p spin eigenfunctions (8)-(10), one can derive spin free working expressions for the matrix elements pertaining to the different blocks of the matrix. For the main 2h1p blocks of the Hamiltonian matrix, one obtains

1. $\mathbf{M}_{S_{1} S_{1}}$

$$
\begin{aligned}
\left\langle\Psi_{a k l}^{S_{1}}\left|\hat{H}-E_{0}\right| \Psi_{a k^{\prime} l^{\prime}}^{S_{1}}\right\rangle & =\left(\epsilon_{a}-\epsilon_{k}-\epsilon_{l}\right) \delta_{k k^{\prime}} \delta_{l l^{\prime}}+\left\langle k l \mid k^{\prime} l^{\prime}\right\rangle+\left\langle k l \mid l^{\prime} k^{\prime}\right\rangle \\
& -\frac{1}{2}(2\langle k a \mid k a\rangle-\langle k a \mid a k\rangle) \delta_{k k^{\prime}} \delta_{l l^{\prime}} \\
& -\frac{1}{2}\left(2\left\langle l a \mid l^{\prime} a\right\rangle-\left\langle l a \mid a l^{\prime}\right\rangle\right) \delta_{k k^{\prime}}
\end{aligned}
$$

2. $\mathbf{M}_{S_{2} S_{2}}$

$$
\left\langle\Psi_{a k k}^{S_{1}}\left|\hat{H}-E_{0}\right| \Psi_{a k^{\prime} k^{\prime}}^{S_{1}}\right\rangle=\left(\epsilon_{a}-2 \epsilon_{k}\right) \delta_{k k^{\prime}}-(2\langle k a \mid k a\rangle-\langle k a \mid a k\rangle) \delta_{k k^{\prime}}+\left\langle k k \mid k^{\prime} k^{\prime}\right\rangle
$$

3. $\mathbf{M}_{T T}$

$$
\begin{aligned}
\left\langle\Psi_{a k l}^{T}\left|\hat{H}-E_{0}\right| \Psi_{a k^{\prime} l^{\prime}}^{T}\right\rangle & =\left(\epsilon_{a}-\epsilon_{k}-\epsilon_{l}\right) \delta_{k k^{\prime}} \delta_{l l^{\prime}}+\left\langle k l \mid k^{\prime} l^{\prime}\right\rangle-\left\langle k l \mid l^{\prime} k^{\prime}\right\rangle \\
& +\left(-\langle a k \mid a k\rangle-\langle a l \mid a l\rangle+\frac{3}{2}\langle a k \mid k a\rangle+\frac{3}{2}\langle a l \mid l a\rangle\right) \delta_{k k^{\prime}} \delta_{l l^{\prime}} \\
& +\left(-\left\langle a l^{\prime} \mid a l\right\rangle+\frac{3}{2}\left\langle a l^{\prime} \mid l a\right\rangle\right) \delta_{k k^{\prime}}\left(1-\delta_{l l^{\prime}}\right)
\end{aligned}
$$

An analogous derivation for the coupling blocks gives the following expressions

1. $\mathbf{C}_{S_{1} S_{2}}$

$$
\left\langle\Psi_{a k l}^{S_{1}}\left|\hat{H}-E_{0}\right| \Psi_{a k^{\prime} k^{\prime}}^{S_{2}}\right\rangle=\sqrt{2}\left\langle k l \mid k^{\prime} k^{\prime}\right\rangle+\frac{1}{\sqrt{2}}(-2\langle a k \mid a l\rangle+\langle a k \mid l a\rangle) \delta_{k k^{\prime}}
$$

2. $\mathbf{C}_{S_{1} T}$

$$
\left\langle\Psi_{a k l}^{S_{1}}\left|\hat{H}-E_{0}\right| \Psi_{a k^{\prime} l^{\prime}}^{T}\right\rangle=\frac{\sqrt{3}}{2}\left\langle a l^{\prime} \mid l a\right\rangle \delta_{k k^{\prime}}-\frac{\sqrt{3}}{2}\langle a k \mid k a\rangle \delta_{k k^{\prime}} \delta_{l l^{\prime}}
$$

3. $\mathbf{C}_{S_{2} T}$

$$
\left\langle\Psi_{a k k}^{S_{2}}\left|\hat{H}-E_{0}\right| \Psi_{a k^{\prime} l^{\prime}}^{T}\right\rangle=\frac{\sqrt{3}}{2}\left\langle a l^{\prime} \mid k a\right\rangle \delta_{k k^{\prime}}
$$


Finally, after diagonalisation of the Hamiltonian matrix for a fixed virtual orbital, the final states are obtained as a linear combination of the spin-adapted 2h1p Hartree-Fock configurations

$$
\left|\tilde{\chi}_{q}^{a}\right\rangle=\sum_{k l} C_{a k l}^{q}\left|\Psi_{a k l}^{X}\right\rangle, X=\left\{S_{1}, S_{2}, T\right\}
$$

The coupling elements Eq. (11) used in the Stieltjes procedure can then be obtained as linear combinations of the coupling elements of the individual spin-adapted 2h1p Hartree-Fock configurations multiplied by the coefficients obtained upon diagonalisation of the Hamiltonian. To this end, one needs the coupling elements of the individual spin-adapted 2h1p HartreeFock configurations, which are the Wigner-Weisskopf expressions for the partial widths of the electronic decay process [71]

$$
\begin{aligned}
\left\langle\Psi_{a k l}^{S_{1}}\left|\hat{H}-E_{0}\right| \Psi_{i}\right\rangle & =\frac{1}{\sqrt{2}}(\langle a i \mid l k\rangle+\langle a i \mid k l\rangle) \\
\left\langle\Psi_{a k k}^{S_{2}}\left|\hat{H}-E_{0}\right| \Psi_{i}\right\rangle & =\langle a i \mid k k\rangle \\
\left\langle\Psi_{a k l}^{T}\left|\hat{H}-E_{0}\right| \Psi_{i}\right\rangle & =\sqrt{\frac{3}{2}}(\langle a i \mid l k\rangle-\langle a i \mid k l\rangle)
\end{aligned}
$$

[1] U. Fano, Phys. Rev. 124, 1866 (1961).

[2] N. Moiseyev, Non-Hermitian Quantum Mechanics (Cambridge University Press, 2011), ISBN 9780511976186.

[3] H. Feshbach, Rev. Mod. Phys. 36, 1076 (1964).

[4] P. Auger, J. Phys. Radium 6, 205 (1925).

[5] G. Howat, T. Åberg, and O. Goscinski, J. Phys. B At. Mol. Opt. Phys. 11, 1575 (1978).

[6] J. Schirmer, L. S. Cederbaum, and O. Walter, Phys. Rev. A 28, 1237 (1983).

[7] D. Danovich, Wiley Interdisciplinary Reviews: Computational Molecular Science 1, 377 (2011).

[8] F. Tarantelli, A. Sgamellotti, L. S. Cederbaum, and J. Schirmer, J. Chem. Phys. 86, 2201 (1987).

[9] F. Tarantelli, A. Sgamellotti, and L. S. Cederbaum, J. Chem. Phys. 94, 523 (1991).

[10] L. S. Cederbaum and F. Tarantelli, J. Chem. Phys. 98, 9691 (1993). 
[11] Y.-C. Chiang, F. Otto, H.-D. Meyer, and L. S. Cederbaum, Phys. Rev. Lett. 107, 173001 (2011).

[12] N. Sisourat, J. Chem. Phys. 139, 074111 (2013).

[13] L. S. Cederbaum, J. Zobeley, and F. Tarantelli, Phys. Rev. Lett. 79, 4778 (1997).

[14] See http://www.pci.uni-heidelberg.de/tc/usr/icd/ICD.refbase.html for a complete list of ICD publications.

[15] J. Zobeley, R. Santra, and L. S. Cederbaum, J. Chem. Phys. 115, 5076 (2001).

[16] K. Gokhberg and L. S. Cederbaum, J. Phys. B At. Mol. Opt. Phys. 42, 231001 (2009).

[17] A. Dreuw and S. Faraji, Phys. Chem. Chem. Phys. 15, 19957 (2013).

[18] P. H. P. Harbach, M. Schneider, S. Faraji, and A. Dreuw, J. Phys. Chem. Lett. 4, 943 (2013).

[19] K. Gokhberg, P. Kolorenč, A. I. Kuleff, and L. S. Cederbaum, Nature 505, 661 (2014).

[20] V. Stumpf, K. Gokhberg, and L. S. Cederbaum, Nat. Chem. 8, 237 (2016).

[21] V. Carravetta and H. gren, Phys. Rev. A 35, 1022 (1987).

[22] B. Schimmelpfennig, B. Nestmann, and S. Peyerimhoff, J. Electron Spectrosc. Relat. Phenom. 74, 173 (1995).

[23] R. Colle and S. Simonucci, Phys. Rev. A 39, 6247 (1989).

[24] E. Z. Chelkowska and F. P. Larkins, Atom. Data Nucl. Data 49, 121 (1991).

[25] M. Mitani, O. Takahashi, K. Saito, and S. Iwata, J. Electron Spectrosc. Relat. Phenom. 128, 103 (2003).

[26] R. Santra and L. S. Cederbaum, J. Chem. Phys. 115, 6853 (2001).

[27] N. Vaval and L. S. Cederbaum, J. Chem. Phys. 126, 164110 (2007).

[28] A. Ghosh, S. Pal, and N. Vaval, J. Chem. Phys. 139, 064112 (2013).

[29] A. Ghosh, S. Pal, and N. Vaval, Mol. Phys. 112, 669 (2014).

[30] R. Santra, J. Zobeley, and L. S. Cederbaum, Phys. Rev. B 64, 245104 (2001).

[31] V. Averbukh and L. S. Cederbaum, J. Chem. Phys. 123, 204107 (2005).

[32] A. U. Hazi, in Electron-Molecule and Photon-Molecule Collisions, edited by T. Rescigno, V. McKoy, and B. Schneider (Plenum Press, New York and London, 1979).

[33] P. W. Langhoff, in Electron-Molecule and Photon-Molecule Collisions, edited by T. Rescigno, V. McKoy, and B. Schneider (Plenum Press, New York and London, 1979).

[34] F. Müller-Plathe and G. H. F. Diercksen, Phys. Rev. A 40, 696 (1989).

[35] P. Kolorenč, V. Averbukh, K. Gokhberg, and L. Cederbaum, J. Chem. Phys. 129, 244102 
(2008).

[36] T. Miteva, Y.-C. Chiang, P. Kolorenč, A. I. Kuleff, K. Gokhberg, and L. S. Cederbaum, J. Chem. Phys. 141, 064307 (2014).

[37] T. Miteva, Y.-C. Chiang, P. Kolorenč, A. I. Kuleff, L. S. Cederbaum, and K. Gokhberg, J. Chem. Phys. 141, 164303 (2014).

[38] P. Kolorenč and N. Sisourat, J. Chem. Phys. 143, 224310 (2015).

[39] G. Jabbari, K. Sadri, L. S. Cederbaum, and K. Gokhberg, J. Chem. Phys. 144, 164307 (2016).

[40] K. Kaufmann, W. Baumeister, and M. Jungen, J. Phys. B At. Mol. Opt. Phys. 22, 2223 (1989).

[41] W. L. Jolly and D. N. Hendrickson, J. Am. Chem. Soc. 92, 1863 (1970).

[42] J. M. Hollander and W. L. Jolly, Acc. Chem. Res. 3, 193 (1970).

[43] V. Averbukh and L. S. Cederbaum, J. Chem. Phys. 125, 094107 (2006).

[44] S. Scheit, V. Averbukh, H.-D. Meyer, J. Zobeley, and L. S. Cederbaum, J. Chem. Phys. 124, $154305(2006)$.

[45] P. O'Keeffe, A. Ciavardini, E. Ripani, P. Bolognesi, M. Coreno, L. Avaldi, M. Devetta, M. Di Fraia, C. Callegari, K. C. Prince, et al., Phys. Rev. A 90, 042508 (2014).

[46] A. I. Kuleff and L. S. Cederbaum, Phys. Rev. Lett. 98, 083201 (2007).

[47] M. O. Krause and J. H. Oliver, J. Phys. Chem. Ref. Data 8, 329 (1979).

[48] N. Moiseyev, R. Santra, J. Zobeley, and L. S. Cederbaum, J. Chem. Phys. 114, 7351 (2001).

[49] S. Scheit, V. Averbukh, H.-D. Meyer, N. Moiseyev, R. Santra, T. Sommerfeld, J. Zobeley, and L. S. Cederbaum, J. Chem. Phys. 121, 8393 (2004).

[50] K. Schnorr, A. Senftleben, M. Kurka, A. Rudenko, L. Foucar, G. Schmid, A. Broska, T. Pfeifer, K. Meyer, D. Anielski, et al., Phys. Rev. Lett. 111, 093402 (2013).

[51] T. Jahnke, A. Czasch, M. S. Schöffler, S. Schössler, A. Knapp, M. Käsz, J. Titze, C. Wimmer, K. Kreidi, R. E. Grisenti, et al., Phys. Rev. Lett. 93, 163401 (2004).

[52] R. Santra, J. Zobeley, L. S. Cederbaum, and N. Moiseyev, Phys. Rev. Lett. 85, 4490 (2000).

[53] P. Kolorenč and V. Averbukh, J. Chem. Phys. 135, 134314 (2011).

[54] A. Albiez, M. Thoma, W. Weber, and W. Mehlhorn, Z. Phys. D 16, 97 (1990).

[55] L. Avaldi, G. Dawber, R. Camilloni, G. C. King, M. Roper, M. R. F. Siggel, G. Stefani, M. Zitnik, A. Lisini, and P. Decleva, Phys. Rev. A 51, 5025 (1995).

[56] T. H. Dunning, J. Chem. Phys. 90, 1007 (1989). 
[57] D. E. Woon and T. H. Dunning, Jr., J. Chem. Phys. 98, 1358 (1993).

[58] The exponents of the diffuse $s^{-}, p$ - and $d$-functions ranged between 100.0 and 1.0. The exponents were obtained according to the relation $\xi_{k}=\xi_{1} \beta^{\frac{k-1}{n-1}}$, where $\xi_{1}=100.0, \beta=0.01$ and $n$ is the number of even tempered basis functions.

[59] C. D. Wagner, Faraday Discuss. Chem. Soc. 60, 291 (1975).

[60] M. W. Schmidt, K. K. Baldridge, J. A. Boatz, S. T. Elbert, M. S. Gordon, J. H. Jensen, S. Koseki, N. Matsunaga, K. A. Nguyen, S. Su, et al., J. Comp. Chem. 14, 1347 (1993).

[61] T. H. Dunning, J. Chem. Phys. 90, 1007 (1989).

[62] R. A. Aziz and M. Slaman, Chem. Phys. 130, 187 (1989).

[63] J.-U. Grabow, A. S. Pine, G. T. Fraser, F. J. Lovas, R. D. Suenram, T. Emilsson, E. Arunan, and H. S. Gutowsky, J. Chem. Phys. 102, 1181 (1995).

[64] D. Griffin, D. M. Mitnik, and N. R. Badnell, J. Phys. B At. Mol. Opt. Phys. 34, 4401 (2001).

[65] G. Marr and J. West, Atomic Data and Nuclear Data Tables 18, 497 (1976).

[66] A. Kramida, Y. Ralchenko, J. Reader, and N. A. Team, NIST Atomic Spectra Database (version 5.1) (National Institute of Standards and Technology, Gaithersburg, MD) (2013), URL http://physics.nist.gov/asd.

[67] V. Averbukh, I. B. Müller, and L. S. Cederbaum, Phys. Rev. Lett. 93, 263002 (2004).

[68] U. Fano and J. W. Cooper, Rev. Mod. Phys. 40, 441 (1968).

[69] J. Matthew and Y. Komninos, Surf. Sci. 53, 716 (1975).

[70] J. Schirmer, A. B. Trofimov, and G. Stelter, J. Chem. Phys. 109, 4734 (1998).

[71] R. Santra and L. S. Cederbaum, Phys. Rev. 368, 1 (2002). 
File(s) excluded from PDF

The following file(s) will not be converted:

FanocI_final.tex

fig1_ne_auger.eps

fig2_nene_icd_sigmau.eps

fig3_near_icd.eps

fig4_nene_icd_r_qz_7s7p7d.eps

Please click 'Download zip file' to download the most recent files related to this submission. 